\title{
Towards Improving Process Control in Sheet Metal Forming: A Hybrid Data- and Model-Based Approach
}

\author{
Sravan TATIPALA ${ }^{\mathrm{a}, 1}$, Johan WALL ${ }^{\mathrm{a}}$, Tobias LARSSON ${ }^{\mathrm{a}}$, Christian JOHANSSON ${ }^{\mathrm{a}}$, \\ and Mats SIGVANT ${ }^{\mathrm{a}, \mathrm{b}}$ \\ ${ }^{a}$ Department of Mechanical Engineering, Blekinge Institute of Technology, Sweden \\ ${ }^{\mathrm{b}}$ Volvo Cars, Dept. 81153 Stamping CAE \& Die Development, Sweden
}

\begin{abstract}
Ability to predict and control involved parameters and hence the outcome of sheet metal forming processes demand holistic knowledge of the product/-process parameter influences and their contribution in shaping the output product quality. Recent improvements in the ability to harvest inline production data and the capability to understand complex process behaviour through computer simulations opens up the possibility for new monitoring and control approaches for improving production process performance and output product quality. Current work presents a framework for monitoring and control of sheet metal forming processes which incorporates a hybrid data-and model-based approach. An initial attempt to evaluate the proposed frameworks' ability to support output product quality and process performance enhancements is made by implementing the proposed approach via an in-house built wire-bending machine prototype. Initial experiments conducted using the built prototype indicate that the proposed framework has the potential to support such enhancements and further work is needed to validate the overall framework.
\end{abstract}

Keywords. Industry 4.0, modelling, simulation, process control, smart manufacturing

\section{Introduction}

In the past, most industrial process monitoring (IPM) approaches were focused on fault detection i.e., the ability to detect a fault and to reduce the time between a faults' occurrence and detection [1]. The identified faults were then commonly mitigated in an ad-hoc manner through trial and error based on the expertise of the machine operators on-site. More recently, with concepts like zero-defect manufacturing gaining importance, the focus has shifted towards fault diagnosis and troubleshooting activities that consume a considerably larger portion of the process downtime [1,2] compared to fault detection activities. In this context, several data-driven [1,3-6], model-based [7-9] and statistical [10] approaches have been proposed to support the identification of the underlying root cause of a fault. However, most of these approaches lack features necessary to completely diagnose and isolate a fault [11]. Similarly, numerous control approaches have been developed to regulate increasingly complex manufacturing processes as described in $[12,13]$. Although these approaches have gained significant

\footnotetext{
${ }^{1}$ Corresponding Author. sravan.tatipala@bth.se
} 
popularity within sophisticated manufacturing processes such as in semiconductor production or chemical plants, their potential within other manufacturing domains such as metal forming remain underexploited [13]. Most control approaches within sheet metal forming aim to control and optimize machine parameter settings instead of the product properties which is usually the property of interest for the end customer $[14,15]$. Furthermore, the control actions in such approaches are generally based on either offline models, or on online process models which involve several approximations to be computationally feasible [14]. In either case, the influence of product/-process parameter correlations on the output product quality is overlooked by not explicitly modeling such complex relationships [16]. Therefore, in the presence of changing production conditions, the overall effectiveness of control loops is affected leading to higher costs and process downtime.

Recent improvements in the ability to harvest operation data combined with computationally efficient simulation setups opens up the possibility for newer concepts to control product/-process quality. The dawn of the fourth industrial revolution, commonly denoted as Industry 4.0 [17], promises new opportunities in this regard. A central theme in Industry 4.0 is connectedness enabled via embedded electronics, software, sensors and network connectivity, allowing components within the manufacturing plant to exchange data regarding specific product or process attributes over the internet in order to improve the overall manufacturing efficiency. However, due to the inherent complexity, providing proper context to the gathered data enabling valuable interpretation is both difficult and time-consuming. Efforts towards exploiting operation data, process models and simulations to improve product quality are gaining popularity $[6,8,18]$ and it is believed that the amalgamation of data-driven and modelbased procedures has the potential to provide a predictive dimension to process control in addition to fault detection and diagnostic features $[1,15]$. By combining data-driven and model-based approaches, a proper context can be provided to the harvested data. Computer models and simulations hold potential in this regard which have proved to be a powerful means for gathering deeper insights of complex stochastic processes. Furthermore, the development of demonstration platforms, such as test-beds and prototypes, are crucial to explore the benefits of digitalization [19].

Current work, while building on previously conducted research [20], presents and tests a framework for enhancing monitoring and control of sheet metal forming process through an in-house built prototype. The following sections describe the proposed framework which involves a hybrid data- and model-based approach in addition to the prototype and the initial experiments conducted using it.

\section{A hybrid approach to process monitoring and control}

A hybrid approach to process monitoring and control contains elements that are datadriven as well as model-based. The former refers to approaches that extract necessary information for the underlying process model structure predominantly from operation data (in addition to subsidiary sources such as background process knowledge, process flowsheets, etc.). The latter refers to approaches where the underlying model structure is based on a priori knowledge of the process and its behavior deduced from firstprinciple models incorporating quite detailed process-specific structure. On one hand, data-driven approaches benefit by requiring minimal understanding of the inherent process mechanisms [3] while on the other hand; suffer from dimensionality issues, 
often require huge amounts of data for reliable performance and do not contain features for full fault diagnosis and isolability [1]. Model-based approaches albeit capture and provide a clear cause-effect relationship between product/-process parameters [6] they fail to take into account the external disturbances and noises prevalent in real production processes [3] while also require rigorous experimentation/effort to derive the process models [14].

Depending on the manufacturing process and scenario, different approaches are suitable. In general, data-driven approaches are popular in scenarios where operation data is relatively easy to acquire, and the process involves few variables (and their interdependencies) to monitor/control. However, the reliability of data-driven approaches is strongly influenced by the quantity and quality of acquired data. When the number of product/-process variables increase, using data-driven approaches could lead to ambiguous results since they lack necessary information for full fault investigation and root-cause identification. Accordingly, model-based approaches are preferred in scenarios that require comparatively more information regarding process phenomena and the influences of involved product/-process variables in order to execute control actions. Approaches which involve process models, are often derived from first-principle models or detailed numerical models which demand exceptional computational resources to be useful in real-time making them impractical with the current computing technology [14]. Figure 1 depicts various considerations involved in a model-driven scenario (digital twin) of sheet metal forming process for car body component manufacturing.

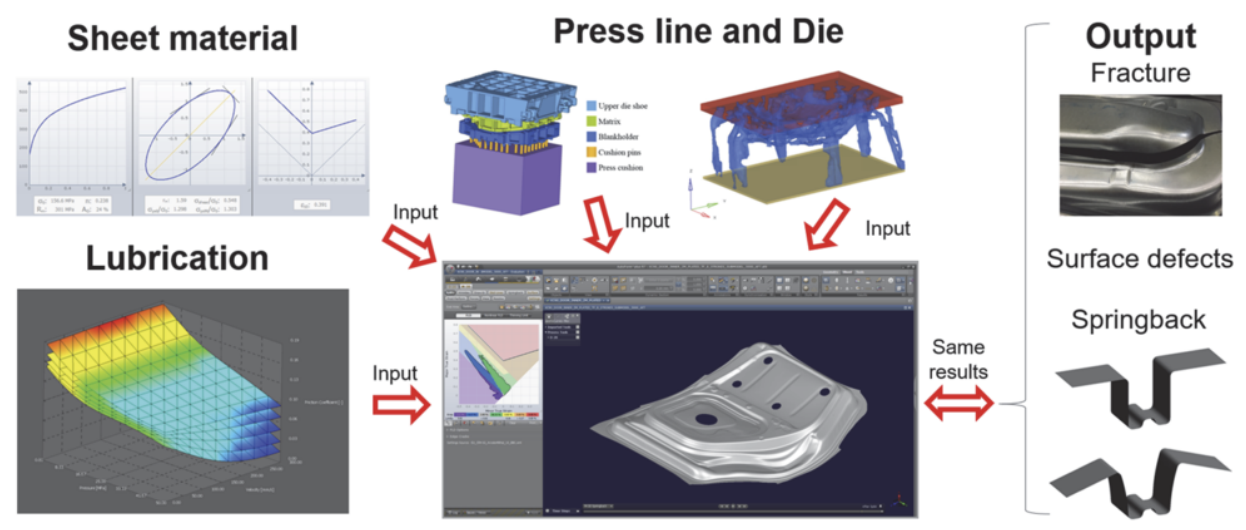

Figure 1. A schematic view of a digital twin of a die and a press line [21].

Due to high computational costs required for solving such models, it is common to develop reduced process models through various strategies. In general, model-based approaches include a better mapping of the cause-effect relationship between product/process variables although the inherent approximations create a mismatch between the model and reality. Moreover, other problems are involved such as, variability in product/-process properties (e.g., temperature, surface properties) that cause variation in process performance are usually not modelled. It is believed that the combination of data-driven methods that capture fluctuations present in reality with model-based approaches that comprise of extensive knowledge regarding product/-process behavior could benefit the monitoring and control approaches in the context of complex manufacturing processes. 


\section{Hybrid framework proposal}

The proposed hybrid framework, combining data-driven and model-based concepts into a hybrid methodology, is presented in Figure 2. The framework consists of the following steps; It starts with an input to the manufacturing line which is uniquely marked. Through that marking, the input is identified, and input specification data is retrieved from data storage (e.g., material properties, surface coating), while other input data (e.g., temperature, thickness, friction conditions), not available from data storage, is measured using sensors in the process. The retrieved and measured data, together with the desired target product specifications is fed into process models to proactively predict the product/-process settings needed to attain the desired output.

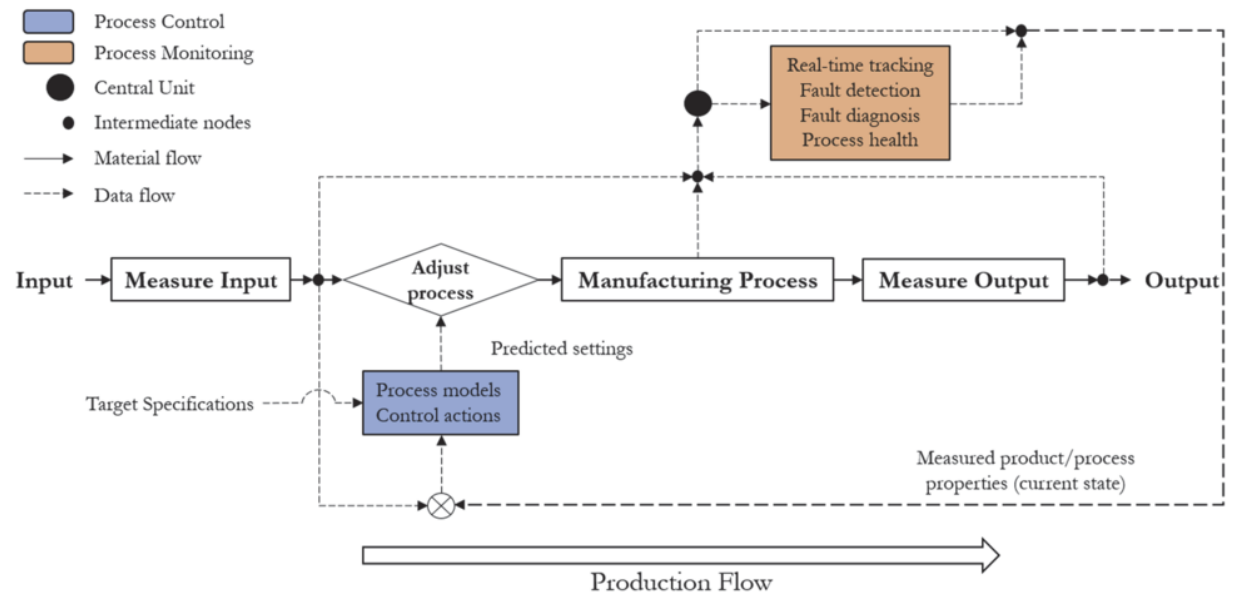

Figure 2. Framework incorporating a hybrid data-and model-based approach.

Available data is used by the monitoring platform to supervise product/-process variables, detect faults, identify root-cause of faults, and to maintain process health. The monitoring platform includes data-driven models (primarily for variable supervision, fault detection, diagnosis and process health monitoring), and contains information about normal operation conditions (NOC). Depending on the scenario, such as; extent of data possible to harvest, data storage/transmission infrastructure, type of process models as well as the effort required to derive them, various datadriven/model-based methods are suitable. The information from the monitoring platform in addition to measured data is fed into the process control system. The control system uses reduced process models (e.g., metamodels [8,9], for viability in real-time) to determine appropriate control response in order to diminish the gap between actual process output and desired process output. These settings are used to adjust the manufacturing process. The framework includes a feed-forward control loop (to adjust process based on input measurements), a feed-back control loop (to adjust process based on in-line and output parameter measurements) as well as a monitoring platform for enhancing product/-process supervision, process fault detection/diagnosis and for providing product/-process current state information to the control system. All process sensors continuously stream data to a central unit for use during different process steps. In addition, intermediate nodes store data immediately required for the 
next stage thus facilitating faster communication between process components and smooth execution of the various stages.

\section{Method}

\subsection{Demonstrator: Wire-bending machine}

In order to obtain an initial understanding, iteratively develop and test the features of the proposed framework in a safe and resource-efficient manner, an in-house demonstrator (or prototype) was built. In relation to the demonstrator, design choices were guided by aspects such as cost, flexibility, and realizability so that popular engineering tools such as, 3D-printers and open source electronic prototyping platforms could be used to materialize it. Furthermore, it had to include some key elements of a real-industrial setup namely electronics, automation, sensors, control system. Thus, an automated wire-bending machine, inspired from [22], was built. Compared with a press shop manufacturing complex car parts, the proposed demonstrator may be regarded as simplistic, however it is here assumed to include several interesting phenomena such as material non-linearity, springback, vibrations which are also prevalent within realproduction scenarios. To achieve a perfect bend, such aspects need to be controlled. It is believed that the insights gathered from experiments conducted using the demonstrator shall support the implementation of the approach within a real manufacturing setup. Figure 3 shows virtual representation of the demonstrator with its main sections namely, a wire straightening section, a wire feeding section, and a wire bending section.

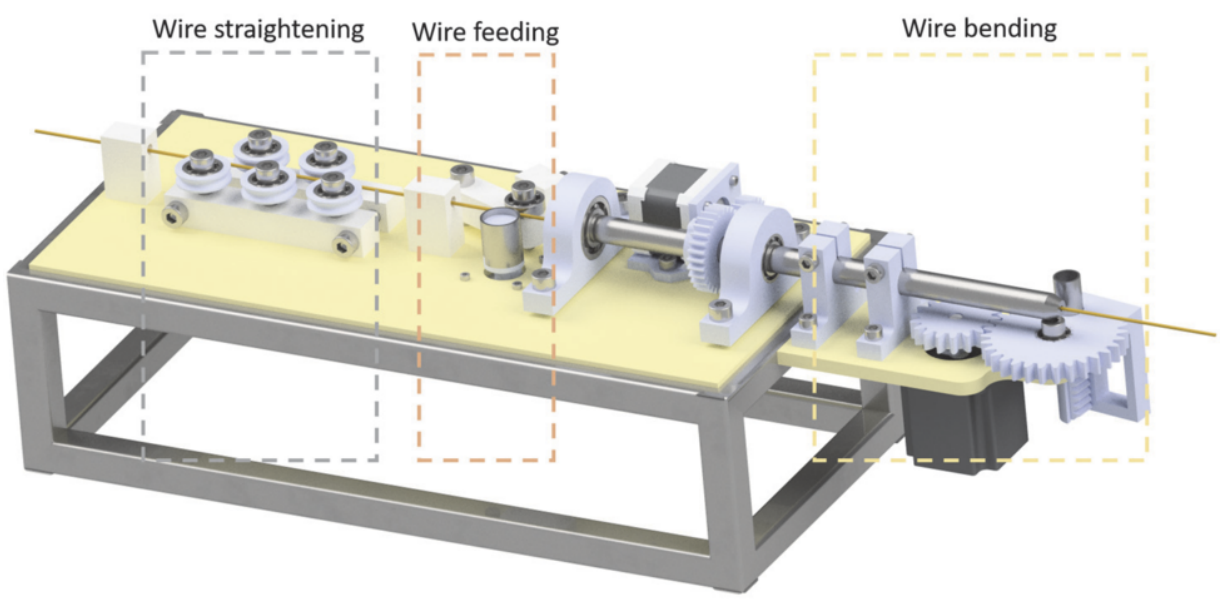

Figure 3. Demonstrator setup.

The basic workflow of the demonstrator is as follows: The wire is fed through a straightening section where the wire is straightened using rollers. Adjacent to it is the feeder section which is responsible for feeding throughout the bending process. The underlying feeding mechanism transforms rotation of a stepper motor into translational feed of the wire. The subsequent bending section involves a rack and pinion 
mechanism and a rotating gear, actuated by a servo motor and a stepper motor, which is used in combination to bend wire to the desired angle.

\subsection{Building reduced process models}

Adapting product/-process settings in response to process measurements demands the ability to measure changes in the system, estimate system status based on measurements and use the system status to predict appropriate system settings. While operation data is measured using sensors in the system, the estimation of the system status and prediction of appropriate settings requires holistic system knowledge. Furthermore, this knowledge needs to be packed into process models that are executable in real-time.

In order to understand the studied system behaviour and the interdependencies/influences of the product/-process parameters on the output product quality, virtual experiments are conducted. For this, a simulation model of the wirebending process is built in a commercial Computer-Aided Engineering (CAE) software aiming to replicate the studied phenomena. The proposed model is validated by comparing the simulated angle of bend with the angle of bend produced from the wire bending machine. The intended parameter space is explored by simulating the behaviour of the system in different scenarios (involving various combinations of system parameter values). This is done in a structured fashion using a pregenerated experimental plan applying an optimal latin hypercube algorithm. To effectively conduct the experiment, an automated strategy inspired from server-client model is adopted as explained in [23].

The results of the simulation experiment revealed that the wire diameter, young's modulus of the material and the angular velocity of the bender motor are the most influential parameters shaping the output bend angle in addition to other subsidiary influences caused by, for example, residual stresses in the material or boundary restraining forces. Further, the results also revealed the presence of springback. Springback is a non-linear phenomenon affecting the dimensional accuracy of the part caused by the recovery of elastic deformation as external bending force is released. A common approach to handle springback is to overbend the part so as to allow the material to springback to the desired bend angle as the elastic part of the deformation is recovered. However, it is a challenging task to understand and determine the extent of overbend required in order to achieve the desired angle of bend without reducing the strength of the part. Results from the conducted simulation experiment, in addition to providing parameter interdependency/influence knowledge, are also used as basis for building metamodels. A metamodel is a mathematical approximation of the response of the original simulation model, hence a metamodel may be described as "a model of the model" [24]. In the context of the proposed hybrid framework, process models need real-time computational performance. Hence, using metamodels is proposed as a remedy since metamodel function calls are generally much faster compared to the original model. However, these approximations introduce errors meaning that such models are usually less accurate in comparison to reality thus potentially amplifying issues such as model uncertainty and related risks. A hybrid approach is believed to potentially mediate for the lack in accuracy by leveraging both model-based and databased insights. Building metamodels involves choosing an approximating model, fitting method and a validation strategy $[25,26]$. The built metamodel is then used for process control as described in the following section. 


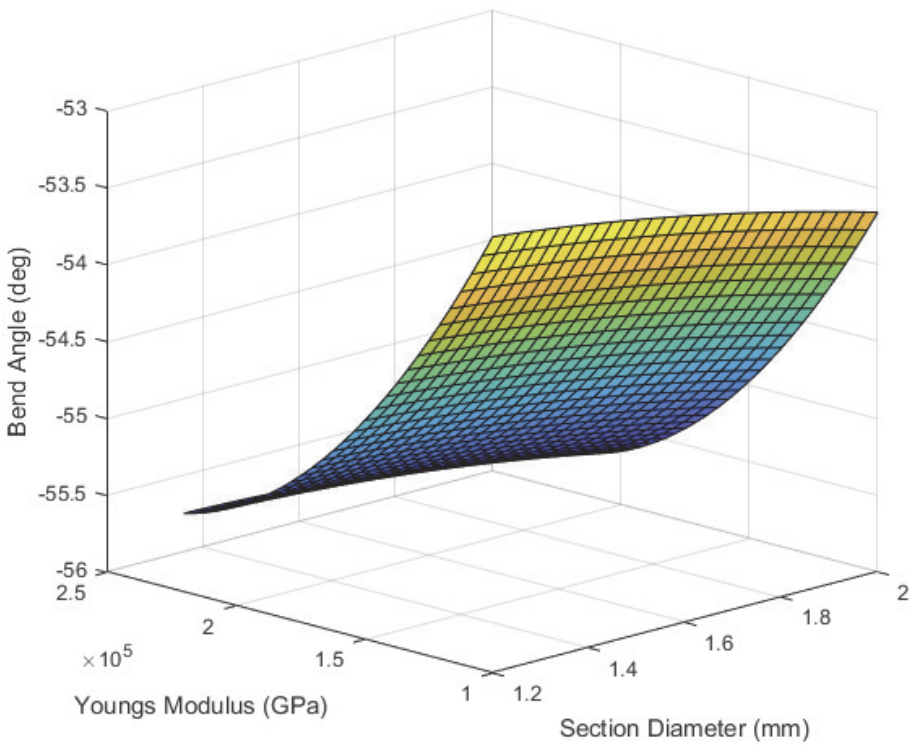

Figure 4. Meta-model presenting the relationship between input variables (young's modulus and wire diameter) with output variable (bend angle).

Figure 4 shows an example of a response surface of the meta-model linking the input variables, young's modulus and wire diameter to the output variable, angle bent. A quadratic polynomial model with three-factor interactions is chosen. The parameters of the polynomial are determined using least square regression analysis by fitting the response surface approximating function to the data collected from the validated simulation model. Several meta-models are built using different combinations of input variables with the output variable.

\section{Results: Prototype in action}

Figure 5 shows a schematic of the prototype (or demonstrator) workflow in relation to the proposed framework. The input to the prototype is a metal wire with an identification tag. In the input measurement station, the tag is scanned to load information regarding the current specimen such as material, dimensions, prework information, coatings, residual stresses, mechanical properties. Additionally, data of the feeder motor rotation is collected to estimate the length of the wire fed forward. Furthermore, the target specifications of the desired output product is loaded. This data is used by the process model to predict appropriate actuator settings. These settings are then used to adjust the machine parameters namely, motor rotation (i.e., the actual amount of rotation required to achieve the desired output bend based on measurements such as dimensions, mechanical properties), motor angular velocity etc., after which the wire bending machine executes its operation. 


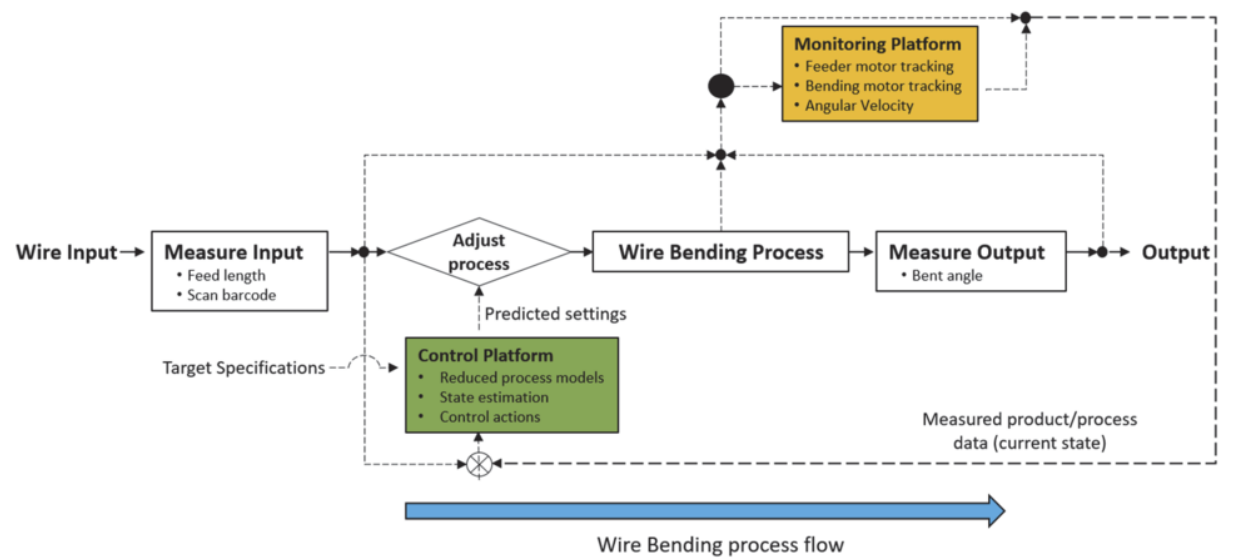

Figure 5. Schematic depicting prototype workflow in relation to the proposed framework.

The monitoring platform continuously collects and logs data during the entire process execution such as bender, feeder and servo motor rotations and notifies when the actuator behaviour deviates from the normal operating condition range. A sensor (3-axis, 6-DOF digital gyroscope and accelerometer sensor [27]) in the output measurement station measures the actual angle of bend. Data from the monitoring platform and output measurement station is fed into the process control platform. Process monitoring and control platform is regulated using a microcontroller board which is programmed to execute an in-house developed algorithm, to facilitate predictions based on reduced process model (metamodels) and to support communication between various sections of the process.

Thus, the metamodels in the process control platform use the current process state to predict appropriate settings for adjusting the process. For instance, the difference between the actual and desired angle of bend is compensated by altering the angular velocity of the bending motor and/or by over-bending or under-bending the wire based on the material young's modulus which influences wire springback. The process is adjusted in every step based on the latest measurement to achieve the desired angle of bend. Continuous process monitoring and measurements help evade process and product quality deviations to go beyond acceptance level. In case, the deviations cross the acceptance level, process fault-diagnosis algorithms help identify the root-cause of the fault which is then fed into the process control platform to prescribe the necessary control action.

In order to evaluate the proposed hybrid approach in comparison with a non-hybrid approach, several experiments were conducted. During these experiments, different combinations of input variables were tested and the output was recorded. Figure 6 shows the output obtained from one of the experiments conducted. In these experiments, wires of different diameters and young's modulus were bent to a desired angle using a hybrid and a non-hybrid approach. The implementation of the hybrid approach is depicted in figure 5, while the implementation of the non-hybrid approach uses nominal system settings similar to a traditional manufacturing process. Since the demonstrator setup is relatively stable in comparison to a real manufacturing setup involving variations due to changes in temperature, friction conditions, material 
properties and rough conditions, random errors were introduced in both cases via the implemented algorithm to see if the errors are compensated for in the consecutive steps.
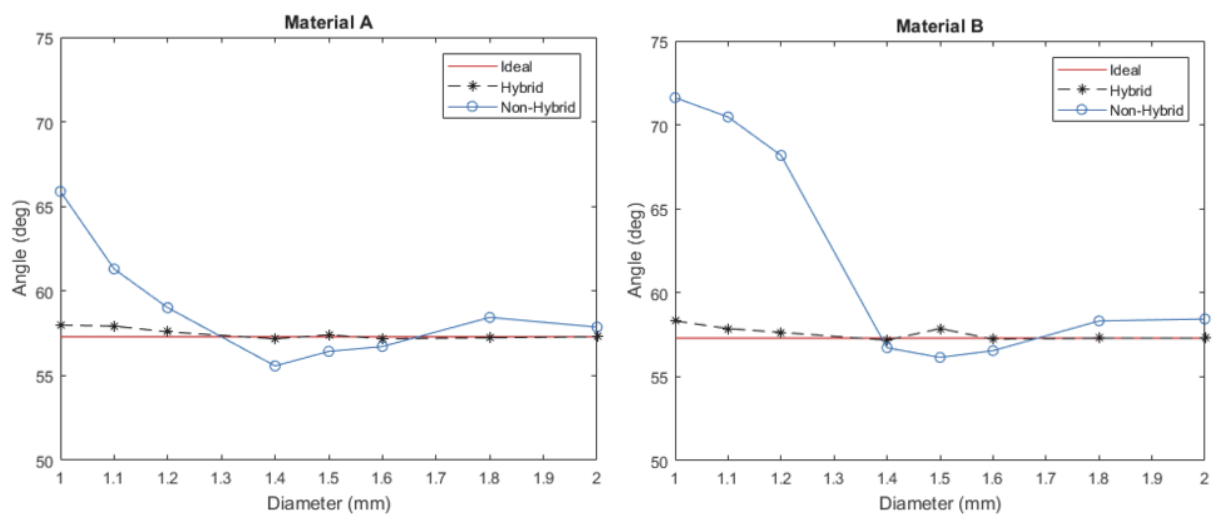

Figure 6. The graph plots the output (actual angle bent) for varying wire diameters, a given desired angle of bend and two different materials showing the comparison with and without a hybrid approach.

Figure 6 plots the actual angle bent (y-axis) for varying wire diameters (x-axis) with respect to a given material (young's modulus) and desired angle of bend (ideal angle). Material A (on left) has a higher young's modulus than Material B (on right). The graphs show that the angle of bend is influenced by factors such as the size of the diameter and material young's modulus. The results show that using a hybrid approach allows to proactively compensate for the wire springback phenomena together with other process errors to consistently produce desired output bend angle.

\section{Discussion and conclusion}

The paper presents a framework for monitoring and control of sheet metal forming process realized through a hybrid data- and model-based approach. An initial attempt to evaluate the proposed frameworks' ability to support product quality and process performance enhancements is made by implementation of the proposed framework via a wire-bending machine demonstrator.

Experimentation with the wire-bending machine shows that the proposed hybrid approach renders an agile process which is capable of handling and automatically adjusting settings in response to process variations caused due to various factors. Although the wire-bending machine demonstrates key aspects of the proposed hybrid approach thus indicating its potential, further work is needed to validate the overall framework. A challenge faced while experimenting with the demonstrator is related to the ability of measuring features of interest within the process. This is even more pronounced in a real-production scenario, for instance, within the sheet metal forming process, due to the prevalence of rough production conditions [28]. Similarly, further work is required for standardizing data transmission, storage and processing tasks within the hybrid approach to facilitate practical application feasibility. In the authors opinion, the proposed approach may form a basis for a digital twin considering the connectivity, sensor setup, process model and data analysis included. However, more analysis tools and a stronger focus on predictive capabilities should be added for it to be a fully functional digital twin. Moving forward into an industry 4.0 enabled 
manufacturing ecosystem where machines and processes shall interact and operate synergistically while allowing digital systems to respond in real-time, a hybrid approach seems promising.

Future work will focus on further development of the framework to include selflearning abilities where process models are updated in-the-loop using machine learning algorithms. Further research would aim to assess the validity of the approach and its implementation on a larger lab-scale production environment. Moving forward, it would also be interesting to explore the ability of the research to support designing products for increasingly smart manufacturing facilities and its potential to benefit the initial stages of the product development.

\section{References}

[1] M. Reis and G. Gins, Industrial process monitoring in the big data/industry 4.0 era: From detection, to diagnosis, to prognosis, Processes, 2017, vol. 5, no. 3.

[2] S. Purr et al., Data-driven inline optimization of the manufacturing process of car body parts, In: IOP Conference Series: Materials Science and Engineering, 2016, vol. 159.

[3] B. Van Stein et al., Towards data driven process control in manufacturing car body parts, In: International Conference on Computational Science and Computational Intelligence (CSCI), 2016, pp. 459-462.

[4] S. Xu, B. Lu, N. Bell, and M. Nixon, Outlier Detection in Dynamic Systems with Multiple Operating Points and Application to Improve Industrial Flare Monitoring, Processes, 2017, vol. 5, no. 2.

[5] X. Xie and R. Schenkendorf, Robust Process Design in Pharmaceutical Manufacturing under Batch-toBatch Variation, Processes, 2017, vol. 7, no. 8.

[6] B. Denkena, M. A. Dittrich, and F. Uhlich, Augmenting milling process data for shape error prediction, In: 49th CIRP Conference on Manufacturing Systems (CIRP-CMS), 2016, vol. 57, pp. 487-491.

[7] J. Son and Y. Du, Model-Based Stochastic Fault Detection and Diagnosis of Lithium-Ion Batteries, Processes, 2019, vol. 7, no. 1.

[8] T. Auerbach et al., Meta-modeling for manufacturing processes, In: International Conference on Intelligent Robotics and Applications, 2011, pp. 199-209.

[9] W. Schulz and T. Al Khawli, Eds., Meta-modelling techniques towards virtual production intelligence, In: Advances in Production Technology, 2015, pp. 69-84.

[10] M. S. Reis, P. M. Saraiva, and B. R. Bakshi, Multiscale statistical process control using wavelet packets , AIChe, 2008, vol. 54, no. 9.

[11] M. S. Reis, G. Gins, and T. J. Rato, Incorporation of process-specific structure in statistical process monitoring: A review, Journal of Quality Technology, 2019, vol. 51, no. 4, pp. 407-421.

[12] D. Djurdjanovic, L. Mears, F. A. Niaki, A. U. Haq, and L. Li, Process and Operations Control in Modern Manufacturing, In: 12th International Manufacturing Science and Engineering Conference, Los Angeles, California, USA, 2017.

[13] D. Djurdjanovic, L. Mears, F. A. Niaki, A. U. Haq, and L. Li, "State of the art review on process, system, and operations control in modern manufacturing, Journal of Manufacturing Science and Engineering, 2018, vol. 140, no. 6.

[14] J. A. Polyblank, J. M. Allwood, and S. R. Duncan, Closed-loop control of product properties in metal forming: A review and prospectus, Journal of Materials Processing Technology, 2014, vol. 214, no. 11, pp. 2333-2348.

[15] S. Duncan, Model based control of product properties: Challenges and Opportunities, In: Forming Technology Forum, 2017, pp. 2-4.

[16] J. Havinga, P. K. Mandal, and T. van den Boogaard, Exploiting data in smart factories: real-time state estimation and model improvement in metal forming mass production, International Journal of Material Forming, 2019, pp. 1-11.

[17] K. Henning, W. D. Lukas, and W. Wahlster, Industrie 4.0: Mit dem Internet der Dinge auf dem Weg zur 4. industriellen Revolution, 2011.

[18] J. Heingärtner, P. Fischer, D. Harsch, Y. Renkci, and P. Hora, Q-Guard-an intelligent process control system, In: Journal of Physics: Conference Series, 2017, vol. 896. 
[19] T. H.-J. Uhlemann, C. Schock, C. Lehmann, and R. Steinhilper, The Digital Twin: Demonstrating the Potential of Real Time Data Acquisition in Production Systems, In: Procedia Manufacturing, 2017, vol. 9, pp. 113-120.

[20] S. Tatipala, J. Wall, C. Johansson, and T. Larsson, A Hybrid Data-Based and Model-Based Approach to Process Monitoring and Control in Sheet Metal Forming, Processes, 2020, vol. 8, no.1.

[21] M. Sigvant, J. Pilthammar, S. Tatipala, and E. Andreasson, Smart Stamping: Improved Quality in Stamping By Model Driven Control, In: Forming Technology Forum, 2018, pp. 59-62.

[22] Dejan, Arduino 3D Wire Bending Machine, How to mechatronics, Accessed: 08.12.2019 [Online]. Available: https://howtomechatronics.com/projects/arduino-3d-wire-bending-machine.

[23] S. Tatipala, J. Wall, C. M. Johansson, and M. Sigvant, Data-driven modelling in the era of Industry 4.0: A case study of friction modelling in sheet metal forming simulations, In: Journal of Physics: Conference Series, 2018, vol. 1063.

[24] J. P. C. Kleijnen, Statistical tools for simulation practitioners, Marcel Dekker, 1987, New York, USA.

[25] T. Simpson, J. Poplinski, P. Koch, and J. K. Allen, Metamodels for Computer-based Engineering Design: Survey and recommendations, Engineering with Computers, 2001, vol. 17, no. 2, pp. 129-150.

[26] R. R. Barton and M. Meckesheimer, Chapter 18: Metamodel-Based Simulation Optimization, In: Supply Chain Management: Design, Coordination and Operation, Elsevier B. V., 2006, pp. 535-574.

[27] Haoyu electronics, GY-521 MPU6050 3-Axis Acceleration Gyroscope 6DOF Module, Accessed: 08.12.2019. [Online]. Available: https://www.hotmcu.com/gy521-mpu6050-3axis-accelerationgyroscope-6dof-module-p-83.html.

[28] S. Maier, A. Liebig, T. Kautz, and W. Volk, Tool-integrated spring back measuring system for automotive press shops, Production Engineering, 2017, vol. 11, no. 3, pp. 307-313. 